\title{
Efficient Simulation of Variability and Heterogeneity in Bioprocess Engineering *
}

\author{
Dennis Pischel ${ }^{*}$ Kai Sundmacher ${ }^{*, * *}$ Robert J. Flassig ${ }^{* *}$ \\ * Otto-von-Guericke-University Magdeburg, Process Systems \\ Engineering, 39106 Magdeburg, Germany \\ ** Max Planck Institute for Dynamics of Complex Technical Systems, \\ Process Systems Engineering, 39106 Magdeburg, Germany
}

\begin{abstract}
Keywords: chemical master equation, Gillespie algorithm, unscented transformation, stochastic differential equation, systems biology.
\end{abstract}

\section{INTRODUCTION}

The individual character of microbial systems is very dominant, since cells vary in plenty of properties, such as morphology, cell cycle state and many more. To accurately capture biological variability by simulations in silico several sources of noise must be considered. In this contribution we refer to intrinsic noise as an inherent stochastic process, extrinsic noise as cell-to-cell variability and external noise as external perturbations, see Fig. 1A. In order to clarify our understanding of the different sources of noise we investigate in Fig. 1B-D their impact on a simple decay process $\mathrm{P} \rightarrow \varnothing$ Pischel et al. (2017). We modeled intrinsic noise via the Gillespie algorithm Gillespie et al. (2013), which captures stochastic biochemical reactions. In contrast extrinsic noise was computed via Monte Carlo sampling of the distributed initial conditions accounting for cell-to-cell variability. Both effects lead to a probability density function describing the abundance of the protein $\mathrm{P}$ for every time point. The synergy of intrinsic and extrinsic noise yields a further spread of the probability density function.

The interaction of different sources of noise and their impact on the overall variability of bioprocesses is hardly investigated due to computational and experimental challenges Lencastre Fernandes et al. (2011); Delvigne and Goffin (2014). A popular approach to model stochastic biochemical reaction systems is by means of the chemical master equation, which governs the temporal evolution of the probability $P$ to find the system in a certain state $\boldsymbol{x}$

$$
\begin{aligned}
\frac{\mathrm{d}}{\mathrm{d} t} P(\boldsymbol{x}(t), t)= & \sum_{k=1}^{m} a_{k}\left(\boldsymbol{x}(t)-\boldsymbol{N}_{k}\right) P\left(\boldsymbol{x}(t)-\boldsymbol{N}_{k}, t\right) \\
& -a_{k}(\boldsymbol{x}(t)) P(\boldsymbol{x}(t), t) .
\end{aligned}
$$

We denote by $\boldsymbol{a}$ the reaction propensities and by $\boldsymbol{N}$ the stoichiometric matrix. The index $k$ indicates the chemical reaction. In general it is not possible to solve the chemical master equation analytically, wherefore approximate methods are used, e.g. the Gillespie algorithm and its derivations, the methods of moments, the system size

\footnotetext{
* This work was supported by the Federal Ministry of Education and Research in Germany [031A304 to D.P.].
}

expansion or the finite state projection algorithm Kazeroonian et al. (2016). All of these methods have several drawbacks and cannot capture different sources of noise. Thus, we present a recent developed method, which is capable to simulate intrinsic, extrinsic and external noise simultaneously Pischel et al. (2017).

\section{EFFICIENT MODELING OF VARIOUS SOURCES OF NOISE}

The simplest approach to model different sources of noise simultaneously is by Monte Carlo sampling of uncertain parameters combined with the temporal system evolution via a stochastic process Wilkinson (2009). In our application the stochastic process is governed by the chemical master equation, which is why we use the Gillespie algorithm through this study. The combined approach is asymptotically exact and yields an accurate solution of the chemical master equation, which goes along with a huge computational load. To accelerate this proceeding we approximate the Monte Carlo sampling of the uncertain parameters by the unscented transformation, which chooses only $2 n_{\sigma}+1$ samples (sigma points) of the $n_{\sigma}$ uncertain parameters deterministically Julier et al. (2000). The sigma points are propagated through time via the $\tau$ leaping algorithm, which is an efficient approximation of the Gillespie algorithm. For every time point $t$ the mean and covariance of the system can be estimated from the propagated sigma points. Since the temporal evolution was computed using a stochastic process this procedure is repeated $n$ times. With assumptions, e.g. normality or log-normality, the underlying distribution $\hat{\boldsymbol{\rho}}_{i}(t)$ can be reconstructed from the mean and covariance for each run $i$. By weighted superposition of the distributions

$$
\tilde{\boldsymbol{\rho}}=\sum_{i=1}^{n} \omega_{i} \boldsymbol{\rho}_{i}=\frac{1}{n} \sum_{i=1}^{n} \hat{\boldsymbol{\rho}}_{i}
$$

we obtain an approximate solution $\tilde{\boldsymbol{\rho}}$ of the chemical master equations with uncertain parameters. This algorithm is outlined in Fig. 2. We applied our method to several examples of systems biology and observed accelerated convergence regarding the statistical moments and the probability density function compared to the combined Monte Carlo approach Pischel et al. (2017). Although our 
A

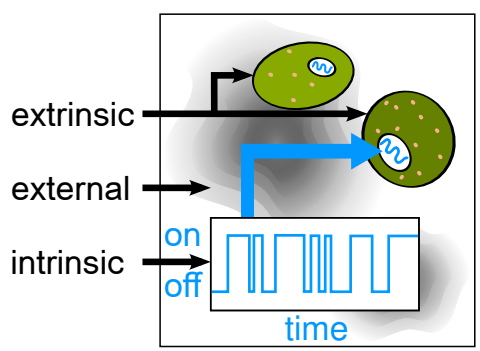

B

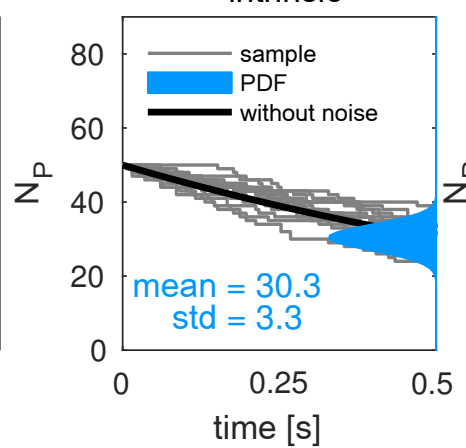

C

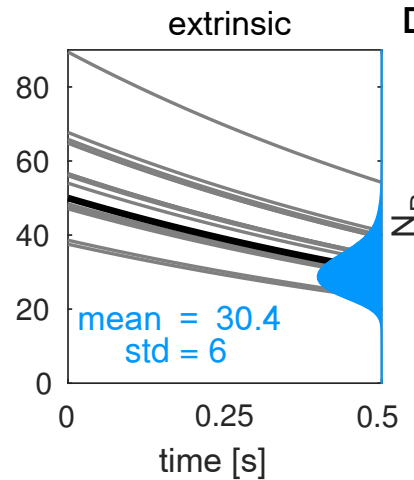

D intrinsic + extrinsic

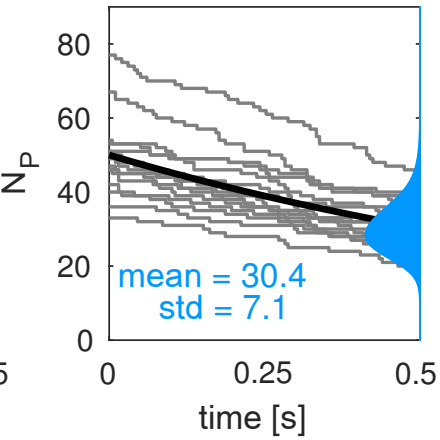

Fig. 1. Noisy biochemical reaction systems: (A) Several sources of noise impact biological variability. Intrinsic (B), extrinsic (C), and intrinsic combined with extrinsic noise perturb a decay process Pischel et al. (2017).

\begin{tabular}{|c|c|}
\hline 1. Selection of $2 n_{\ominus}+1$ sigma points & $\left\{\boldsymbol{\phi}_{i}\right\} \quad$ with $\quad i=0 \ldots 2 n_{\Theta}$ \\
\hline > & M $k=n$ \\
\hline $\begin{array}{l}\text { 2. Temporal evolution of sigma } \\
\text { points through stochastic process via } \\
\text { t-leaping }\end{array}$ & $\left\{\mathbf{y}_{i, k}=\mathbf{h}\left(\phi_{i}, t\right)_{k}\right\}$ \\
\hline & \\
\hline $\begin{array}{l}\text { 3. Calculate } k=1 . . . n \text { sets of } \\
\text { mean } \& \text { covariance of } \boldsymbol{Y} \text { for } \\
\text { each point in time } t\end{array}$ & $\begin{array}{l}\mathbf{E}_{k}(\boldsymbol{Y})=\sum_{i=0}^{2 n_{\ominus}} w_{i} \mathbf{y}_{i, k} \\
\mathbf{C}_{k}(\boldsymbol{Y})=\sum_{i=0}^{2 n_{\ominus}} w_{i}\left(\mathbf{y}_{i, k}-\mathbf{E}_{k}(\boldsymbol{Y})\right)\left(\mathbf{y}_{i, k}-\mathbf{E}_{k}(\boldsymbol{Y})\right)^{\mathrm{T}}\end{array}$ \\
\hline & \\
\hline 4. Assume particular PDF & $\hat{\boldsymbol{\rho}}_{k}(\mathbf{y}) \propto \exp \left(-\frac{1}{2}\left(\mathbf{y}-\mathbf{E}_{k}(\boldsymbol{Y})\right)^{\mathrm{T}} \mathbf{C}_{k}(\boldsymbol{Y})^{-1}\left(\mathbf{y}-\mathbf{E}_{k}(\boldsymbol{Y})\right)\right.$ \\
\hline & \\
\hline 5. Approximate true PDF & $\boldsymbol{\rho}(\mathbf{y}) \approx \sum_{k=1}^{n} \omega_{k} \hat{\boldsymbol{\rho}}_{k}(\mathbf{y}) \quad$ with $\quad \omega_{k}=1 / n$ \\
\hline
\end{tabular}

Fig. 2. Algorithm outline Pischel et al. (2017).

approximate method might in some cases not converge to the exact solution we observe qualitative conformance.

\section{CONCLUSION}

In this study an efficient approach to model different sources of noise in biochemical reaction systems simultaneously was proposed. Our method converges very fast to an approximate solution compared to straightforward Monte Carlo methods. Hence, it is well suited to speed up costly optimization tasks, e.g. parameter estimation problems of distributed, stochastic biochemical systems Pischel et al. (2017). Since optimization of stochastic systems is rarely performed due to its huge computational load Poovathingal and Gunawan (2010); Fröhlich et al. (2016) our approach paves the way to further understanding of uncertainty in complex dynamical systems.

\section{REFERENCES}

Delvigne, F. and Goffin, P. (2014). Microbial heterogeneity affects bioprocess robustness: Dynamic singlecell analysis contributes to understanding of microbial populations. Biotechnology Journal, 9(1), 61-72. doi: 10.1002/biot.201300119.

Fröhlich, F., Thomas, P., Kazeroonian, A., Theis, F., Grima, R., and Hasenauer, J. (2016). Inference for stochastic chemical kinetics using moment equations and system size expansion. PLoS Computational Biology, 12(7). doi:10.1371/journal.pcbi.1005030.

Gillespie, D.T., Hellander, A., and Petzold, L.R. (2013). Perspective: Stochastic algorithms for chemical kinetics. Journal of Chemical Physics, 138(17). doi: $10.1063 / 1.4801941$.

Julier, S., Uhlmann, J., and Durrant-Whyte, H.F. (2000). A new method for the nonlinear transformation of means and covariances in filters and estimators. IEEE Transactions on Automatic Control, 45(3), 477-482. doi: $10.1109 / 9.847726$.

Kazeroonian, A., Fröhlich, F., Raue, A., Theis, F.J., and Hasenauer, J. (2016). Cerena: Chemical reaction network analyzer-a toolbox for the simulation and analysis of stochastic chemical kinetics. PLoS ONE, 11(1), e0146732. doi:10.1371/journal.pone.0146732.

Lencastre Fernandes, R., Nierychlo, M., Lundin, L., Pedersen, A.E., Puentes Tellez, P.E., Dutta, A., Carlquist, M., Bolic, A., Schäpper, D., Brunetti, A.C., Helmark, S., Heins, A.L., Jensen, A.D., Nopens, I., Rottwitt, K., Szita, N., van Elsas, J.D., Nielsen, P.H., Martinussen, J., Sørensen, S.J., Lantz, A.E., and Gernaey, K.V. (2011). Experimental methods and modeling techniques for description of cell population heterogeneity. Biotechnology Advances, 29(6), 575-599. doi: 10.1016/j.biotechadv.2011.03.007.

Pischel, D., Sundmacher, K., and Flassig, R.J. (2017). Efficient simulation of intrinsic, extrinsic and external noise in biochemical systems. Bioinformatics, 33(14), i319-i324. doi:10.1093/bioinformatics/btx253.

Poovathingal, S.K. and Gunawan, R. (2010). Global parameter estimation methods for stochastic biochemical systems. BMC Bioinformatics, 11. doi:10.1186/14712105-11-414.

Wilkinson, D.J. (2009). Stochastic modelling for quantitative description of heterogeneous biological systems. Nature Reviews Genetics, 10(2), 122-133. doi: $10.1038 / \mathrm{nrg} 2509$. 